\title{
The Movement of a Mixture of Cotton with an Air Stream during Pneumatic Transport by Pipeline of Variable Cross Section
}

\author{
Ibrohimjon Tursunov', Nodira Rajapova1, Olimjon Sarimsakov ${ }^{1}$, Botirjon Mardonov ${ }^{2}$ \\ ${ }^{1}$ Department of Technology of Primary Processing of Natural Fibers, Namangan Institute of Engineering and Technology, \\ Namangan, Uzbekistan \\ ${ }^{2}$ Department of Higher Mathematics, Tashkent University of Information Technologies, Tashkent, Uzbekistan \\ Email: olimjon5008@mail.ru
}

How to cite this paper: Tursunov, I., Rajapova, N., Sarimsakov, O. and Mardonov, B. (2019) The Movement of a Mixture of Cotton with an Air Stream during Pneumatic Transport by Pipeline of Variable Cross Section. Engineering, 11, 531-540. https://doi.org/10.4236/eng.2019.118037

Received: June 21, 2019

Accepted: August 26, 2019

Published: August 29, 2019

Copyright (c) 2019 by author(s) and Scientific Research Publishing Inc. This work is licensed under the Creative Commons Attribution International License (CC BY 4.0).

http://creativecommons.org/licenses/by/4.0/

\begin{abstract}
The article considers the movement of a mixture of air and raw cotton through a pipeline with a variable cross-section as a multi-speed heterogeneous medium. The regularities of the movement of components inside the pipeline, the equation of change in the porosity of cotton, air pressure and component velocities in time and along the transportation line are obtained. It was found that in the initial $20-25 \mathrm{~m}$ part of the pneumatic transport pipe there is a sharp decrease in pressure and air flow velocity, while the speed of cotton increases rapidly due to which there is a strong deformation of cotton stretching under the influence of aerodynamic force, which occurs due to the difference in the velocities of the components of the mixture, as a result of which the cotton loosens, and its porosity increases intensively.
\end{abstract}

\section{Keywords}

Air Pressure, Fiber, Pipeline, Porosity, Pneumatic Transportation, Raw Cotton, The Mixture

\section{Introduction}

Depending on the number of components in the pipeline transport, one-component and multi-component media are distinguished. A single-component medium is a medium consisting of a single material or substance, which is called a homogeneous mixture, and a multi-component-of several materials or substances that differ in physical, mechanical, chemical and other properties, is called a heterogeneous mixture [1] [2]. The mathematical description of the motion of a 
multicomponent medium is complicated by the difference in the reaction of each component to the movement of another. Therefore, to simplify the process of theoretical consideration, multicomponent media are taken as a one-component or two-component medium consisting of a continuous phase-air and a discrete phase-material, which ultimately gives more general and less accurate results.

Especially, when considering the processes of pneumatic transportation of materials, including raw cotton, the theory of motion of a discrete medium in a stationary or moving continuous medium has been adopted to date, which will give a more or less distorted idea of the process of moving air and material inside the pipeline [3]. More suitable, in our opinion, to study the process of pneumatic transportation theory, is the theory of multi-speed systems, according to which, all components are moved through the pipeline separately, with interdependent parameters, including speed, which is a new approach to the study of the processes of pneumatic transportation of materials. To ensure the universality of the laws, it should consider the motion of the mixture inside the pipeline with a variable cross section [4] [5].

The mixture of air with fiber particles is assumed to be a heterogeneous mixture, to describe the motion of which you can use the theory of multi-speed systems proposed in the work of Kh. A. Rakhmatullin [6].

Heterogeneous mixtures, as a rule, are described by a multi-speed model taking into account the dynamic effects arising due to the mismatch of the speeds of the individual phases. At the same time, we consider the air to be an ideal fluid and the internal force of interaction is determined through the normal pressure, which is common to the whole mixture, opposite to the direction of movement of the particles of the fibrous medium [7] [8].

The following designations are used in the research:

$u_{0}(x)$-air velocity (index-0) in an arbitrary section of the pipeline;

$u_{1}(x)$-the speed of the particles of raw cotton (index-1) in an arbitrary section of the pipeline;

$u_{00}$-air flow velocity in the pipeline section $x=0$;

$\rho_{0}$-reduced air density in an arbitrary section of the pipeline, $\mathrm{kg} / \mathrm{m}^{3}$;

$\rho_{0}^{(0)}$ - the true density of air, $\mathrm{kg} / \mathrm{m}^{3}$;

$\rho_{00}$-values of air density in section $x=0$;

$\rho_{1}$-the reduced density of the fibrous mass in an arbitrary section of the pipeline, $\mathrm{kg} / \mathrm{m}^{3}$;

$\rho_{1}^{(0)}$ - their true density of fibrous mass, $\mathrm{kg} / \mathrm{m}^{3}$;

$\rho_{10}$-values of the density of the fibrous mass in the cross section;

$\rho_{0}(x), \rho_{1}(x)$ and $u_{0}(x), u_{1}(x)$-current density and velocity values of the components;

$m$-the porosity of cotton in an arbitrary section of the pipe;

$m_{0}$-porosity of cotton in section $x=0$;

$k$-coefficient of resistance of cotton to air movement, $\mathrm{kg} / \mathrm{s}$;

$k_{n}$-the boundary value of the coefficient, $\mathrm{kg} / \mathrm{s}$; 
$p_{0}$-the pressure of the mixture in the cross section $x=0$;

$q=k_{0} p$-lateral flow pressure acting on the walls of the pipeline, $\mathrm{PA}$;

$k_{0}$-lateral pressure coefficient;

$F_{t r}=f q L$ - the friction force between the raw and the surface of the pipe, N;

$f$-the coefficient of friction between the outside surface of the pipe;

$L$-length of the pipe cross-section contour), $\mathrm{m}$.

\section{Study of the Motion of a Multicomponent Medium}

Set the origin of the coordinates in the initial section of the pipeline. We direct the axis along the axis of the pipeline, the cross-sectional area of which varies according to the law, considering the process stationary, denoted by, respectively, the air velocity (index-) and cotton particles of raw mass (index-1) in an arbitrary section of the pipeline. We believe that in the cross section of the pipeline the air flow with speed acts on the moving mass of the mixture. The equations of one-dimensional motion of the components of the mixture and the laws of mass conservation, according to, are written in the form

$$
\begin{gathered}
\rho_{0} u_{0} s \frac{\mathrm{d} u_{0}}{\mathrm{~d} x}=-\frac{\rho_{0}}{\rho_{0}^{(0)}} \frac{\mathrm{d}(s p)}{\mathrm{d} x}+s k\left(u_{1}-u_{0}\right) \\
\rho_{1} u_{1} s \frac{\mathrm{d} u_{1}}{\mathrm{~d} x}=-\frac{\rho_{1}}{\rho_{1}^{(0)}} \frac{\mathrm{d}(s p)}{\mathrm{d} x}-s k\left(u_{1}-u_{0}\right) \\
\rho_{0} u_{0} s=u_{00} \rho_{00} s_{0}=\text { const }, \quad \rho_{1} u_{1} s=u_{10} \rho_{10} s_{0}=\text { const } \\
\rho_{0}=m \rho_{0}^{(0)}, \quad \rho_{1}=(1-m) \rho_{1}^{(0)} \\
\rho_{0}=\frac{m}{m_{0}} \rho_{00}, \quad \rho_{1}=\frac{1-m}{1-m_{0}} \rho_{10}
\end{gathered}
$$

From (3) the following relations follow

$$
u_{0}=\frac{m_{0}}{m} \frac{s_{0}}{s} u_{00}, \quad u_{1}=\frac{1-m_{0}}{1-m} \frac{s_{0}}{s} u_{10}, \frac{\rho_{0}^{(0)}}{\rho_{0}}+\frac{\rho_{1}^{(0)}}{\rho_{1}}=1
$$

Equations (1) and (2) we bring to the form

$$
\begin{gathered}
\rho_{0}^{(0)} u_{0} s \frac{\mathrm{d} u_{0}}{\mathrm{~d} x}=-\frac{\mathrm{d}(s p)}{\mathrm{d} x}+\frac{\rho_{0}^{(0)}}{\rho_{0}} s k\left(u_{1}-u_{0}\right) \\
\rho_{1}^{(0)} u_{1} s \frac{\mathrm{d} u_{1}}{\mathrm{~d} x}=-\frac{\mathrm{d}(s p)}{\mathrm{d} x}-\frac{\rho_{1}^{(0)}}{\rho_{1}} s k\left(u_{1}-u_{0}\right)
\end{gathered}
$$

Excluding the derivative from the system $\frac{\mathrm{d}(s p)}{\mathrm{d} x}$, we get

$$
\rho_{0}^{(0)} u_{0} \frac{\mathrm{d} u_{0}}{\mathrm{~d} x}-\rho_{1}^{(0)} u_{1} \frac{\mathrm{d} u_{1}}{\mathrm{~d} x}=\left(\frac{\rho_{0}^{(0)}}{\rho_{0}}+\frac{\rho_{1}^{(0)}}{\rho_{1}}\right) k\left(u_{1}-u_{0}\right)
$$

Equations (3) and (4) imply that the densities and velocities of the components in an arbitrary section of the pipeline are expressed in terms of porosity 
$m(x)$, which after excluding speeds $u_{0}(x), u_{1}(x)$ from Equation (7) with the help of relations (4), satisfies the equation:

$$
\frac{\mathrm{d} m}{\mathrm{~d} x}=\frac{F_{1}(m)}{F_{0}(m)} \frac{s^{\prime}(x)}{s(x)}+\frac{F_{2}(m)}{F_{0}(m)} \frac{s_{0}}{s(x)}
$$

where:

$$
\begin{gathered}
F_{0}=\rho_{0}^{(0)} u_{00}^{2} m_{0}^{3}(1-m)^{3}+\rho_{1}^{(0)} u_{10}^{2} m^{3}\left(1-m_{0}\right)^{3}, \\
F_{1}=-m(1-m)\left[\rho_{0}^{(0)} u_{00}^{2} m_{0}^{2}(1-m)^{2}-\rho_{1}^{(0)} u_{10}^{2} m^{2}\left(1-m_{0}\right)^{2}\right], \\
F_{1}=m^{2}(1-m)^{2} k\left[u_{00} m_{0}(1-m)-u_{10} m\left(1-m_{0}\right)\right]
\end{gathered}
$$

The pressure $p(x)$ is determined by the formula

$$
p=\frac{s_{0}}{s}\left[p_{0}+u_{00}^{2} \rho_{00}\left(1-\frac{m_{0}}{m} \frac{s_{0}}{s}\right)+u_{10}^{2} \rho_{10}\left(1-\frac{1-m_{0}}{1-m} \frac{s_{0}}{s}\right)\right]
$$

The densities $\rho_{0}(x), \rho_{1}(x)$ and velocities of the components $u_{0}(x), u_{1}(x)$ are expressed in terms of porosity by the Formulas (3) and (4).

In the process of transporting the mixture on the inner wall from the side of the particles of the solid component (cotton particles of raw) acts lateral pressure $q=k_{0} p$ ( $k_{0}$-coefficient of lateral pressure), which in the direction of motion creates a frictional force $f_{q L}$ ( $f$-coefficient of friction between the raw and the surface of the pipe, $L=l(x)$-the length of the contour of the cross-section of the pipe), determined, for example, by the law of dry friction Kulon. In this case, Equations (1) and (2) are written in the form:

$$
\begin{gathered}
\rho_{0} u_{0} s \frac{\mathrm{d} u_{0}}{\mathrm{~d} x}=-\frac{\rho_{0}}{\rho_{0}^{(0)}} \frac{\mathrm{d}(s p)}{\mathrm{d} x}+s k\left(u_{1}-u_{0}\right) \\
\rho_{1} u_{1} s \frac{\mathrm{d} u_{1}}{\mathrm{~d} x}=-\frac{\rho_{1}}{\rho_{1}^{(0)}} \frac{\mathrm{d}(s p)}{\mathrm{d} x}-s k\left(u_{1}-u_{0}\right)-k_{0} l f p(1-m)
\end{gathered}
$$

After excluding the derivative $\frac{\mathrm{d}(s p)}{\mathrm{d} x}$ from system (1) and (11), we obtain

$$
\rho_{0}^{(0)} u_{0} \frac{\mathrm{d} u_{0}}{\mathrm{~d} x}-\rho_{1}^{(0)} u_{1} \frac{\mathrm{d} u_{1}}{\mathrm{~d} x}=k\left(u_{1}-u_{0}\right)+\frac{\rho_{1}^{(0)}}{s \rho_{1}}(1-m) l k_{0} f p
$$

After the statement of the expressions of derivatives $\frac{\mathrm{d} u_{0}}{\mathrm{~d} x}$ and $\frac{\mathrm{d} u_{1}}{\mathrm{~d} x}$ taking into account dependencies (4), we obtain

$$
\begin{gathered}
\frac{\mathrm{d} m}{\mathrm{~d} x}=Z(m, x, p) \\
Z(m, x, p)=\frac{F_{1}(m)}{F_{0}(m)} \frac{s^{\prime}(x)}{s(x)}+\frac{F_{2}(m)}{F_{0}(m)} \frac{s_{0}}{s(x)}-\frac{k_{0} f l(x) s(x) m^{3}(1-m)^{3}}{s_{0}^{2}} p
\end{gathered}
$$

To determine the derivative $\frac{\mathrm{d}(s p)}{\mathrm{d} x}$ we add Equations (10) and (11) and, taking into account (3), we have 


$$
\rho_{00} u_{00} \frac{\mathrm{d} u_{0}}{\mathrm{~d} x}+\rho_{10} u_{10} \frac{\mathrm{d} u_{1}}{\mathrm{~d} x}=-\frac{\mathrm{d} p}{\mathrm{~d} x}-l k_{0} f p(1-m) / s
$$

Adding the values of the derivatives $\frac{\mathrm{d} u_{0}}{\mathrm{~d} x}$ and $\frac{\mathrm{d} u_{1}}{\mathrm{~d} x}$ we get:

$$
\frac{\mathrm{d} p}{\mathrm{~d} x}+Z_{0}(m, x) p=Z_{1}(x, m, p)
$$

Here

$$
\begin{aligned}
& Z_{0}=\frac{l(x) k_{0} f(1-m)}{s(x)}, Z_{1}(x, m, p)=F_{3}(x, m, p)+F_{4}(x, m), \\
& F_{3}=\frac{s_{0}^{2}}{s^{2}(x)} \frac{Z(x, m, p)}{m^{2}(1-m)^{2}}\left[\rho_{00} u_{00}^{2} m_{0}^{2}(1-m)-\rho_{10} u_{10}^{2}\left(1-m_{0}\right)^{2} m\right], \\
& F_{4}=\frac{s_{0}^{2}}{s^{2}(x)} \frac{s^{\prime}(x)}{s(x)} \frac{1}{m(1-m)}\left[\rho_{00} u_{0}^{2} m_{0}(1-m)+\rho_{10} u_{10}^{2} m\left(1-m_{0}\right)\right]
\end{aligned}
$$

Equations (11) and (12) together describe a system that satisfies the following initial conditions for determining cotton porosity and pressure:

$$
\text { At } x=0, \quad m=m_{0}, \quad p=p_{0}=\rho_{00} u_{00}^{2} / 2 \text {. }
$$

\section{Computer Processing and Analysis of the Obtained Regularities}

The equations are processed by the Maple 2015 program with the following parameters for a constant-section pipeline and the corresponding graphical dependencies are obtained: $m_{0}=0.4, u_{00}=20 \mathrm{~m} / \mathrm{s}, \quad \rho_{00}=1.2 \kappa \Gamma / \mathrm{m}^{3}$, $Q_{0}=10000 \kappa г /$ hour $, \rho_{10}=38 \kappa \kappa / \mathrm{m}^{3}, s=s_{0}=\pi D^{2} / 4, \quad l=\pi D, k_{0}=0.6$. The current coefficient of porosity of cotton is equal $m=0.6$, and the boundary values of the coefficient of interaction are respectively equal $k_{n}=50 \mathrm{Hc} / \mathrm{m}^{4}$, $k_{n}=100 \mathrm{Hc} / \mathrm{M}^{4}, k_{n}=200 \mathrm{Hc} / \mathrm{M}^{4}, k_{n}=300 \mathrm{Hc} / \mathrm{m}^{4} \quad$ [9] [10].

Figures 1-4 present the results of an analysis of the equations for the diameter of the pipeline $D=0.4 \mathrm{M}$ : the change in the porosity of the cotton $m(x)$, the velocity of the components $u_{0}(x), u_{1}(x)$, and the pressure $p(x)$ along the length of the pipeline.

To establish the influence of the friction coefficient $f$ on the transportation process, the calculations were made for its various values. In the absence of friction, the movement of the transported material occurs at all values of the interaction coefficient large from zero $(k>0)$. And, in the presence of friction, the movement occurs at $k \geq k_{n}$ ( $k_{n}$-boundary value of the coefficient). At smaller values of this coefficient is $k$. At smaller values of this coefficient $\left(k<k_{n}\right)$ in some sections of the pipeline, the pressure breaks down and movement will not occur. For such cases, the adopted model does not work. For all other cases, the equations obtained correctly describe the process of moving a two-phase flow.

For example, with $f=0.05, f=0.1, f=0.2$ and $f=0.3$, the interaction coefficient will have the following values: $k_{n}=8.7 \mathrm{Hc} / \mathrm{m}^{4}, k_{n}=16.8 \mathrm{Hc} / \mathrm{m}^{4}$, $k_{n}=35.4 \mathrm{Hc} / \mathrm{M}^{4}, k_{n}=50.5 \mathrm{Hc} / \mathrm{m}^{4}$. 
The analysis shows that the value of porosity (Figure 1), retains the property of a regular total increase for large values $k_{n}$ (the graph on the right) is relatively smaller (the graph on the left) and is about 0.85. At the same time, when $k=400 \mathrm{Hc} / \mathrm{M}^{4}$ the increase is much more intense at the beginning of the process, and after passing through 24 - 25 meters, it remains relatively constant.

On the pressure graph (Figure 2) there is a difference with the initial parameters-the pressure at the beginning of the pipeline is about $75 \mathrm{~Pa}$ for smaller (graph on the left), $95 \mathrm{~Pa}$ (graph on the right) for larger values $k_{n}$. However, the pressure value at lower values $k_{n}$ in the initial 1 - 2 meters of the pipeline decreases sharply. When reaching 20 meters the intensity drops, then the pressure is relatively stabilized. At high values $k_{n}$, the pressure decreases with almost the same intensity.

The graph of air velocity (Figure 3 ) shows the property of decline with the same intensity for all values $k_{n}$. When it reaches $50 \mathrm{~m}$, the air velocity drops to $14-12 \mathrm{~m} / \mathrm{s}$, then almost does not change. And the speed of cotton (Figure 4), starting from the 0th value, gradually increases. And when it reaches $50 \mathrm{~m}$, it is relatively stabilized with a value of $7-8 \mathrm{~m} / \mathrm{s}$.

Then, it remains almost unchanged, and the speed of cotton (Figure 4), on the contrary, is of increasing nature. If you look at the charts, the speed of the cotton in the initial 20 meters of the pipeline length changes dramatically, then more stably. At large values of the interaction coefficient, although at the beginning of the pipeline the speed changes greatly, after passing about $30 \mathrm{~m}$ distance

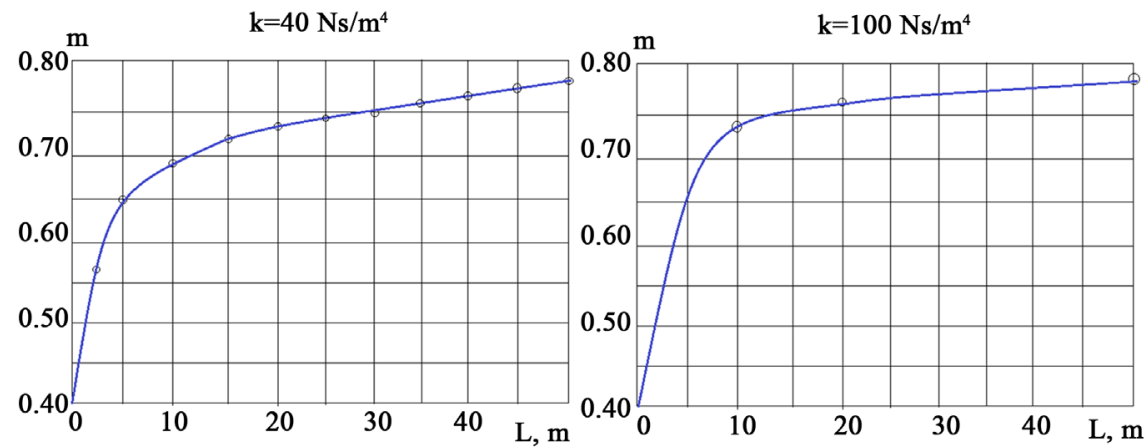

Figure 1. Changing the porosity of cotton along of the pipeline.
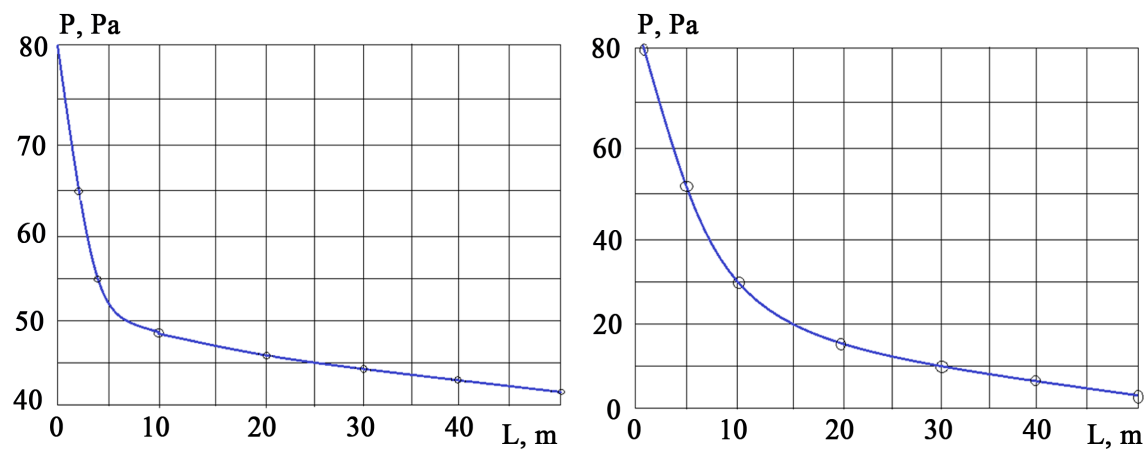

Figure 2. The change in air pressure along the length of the pipeline. 

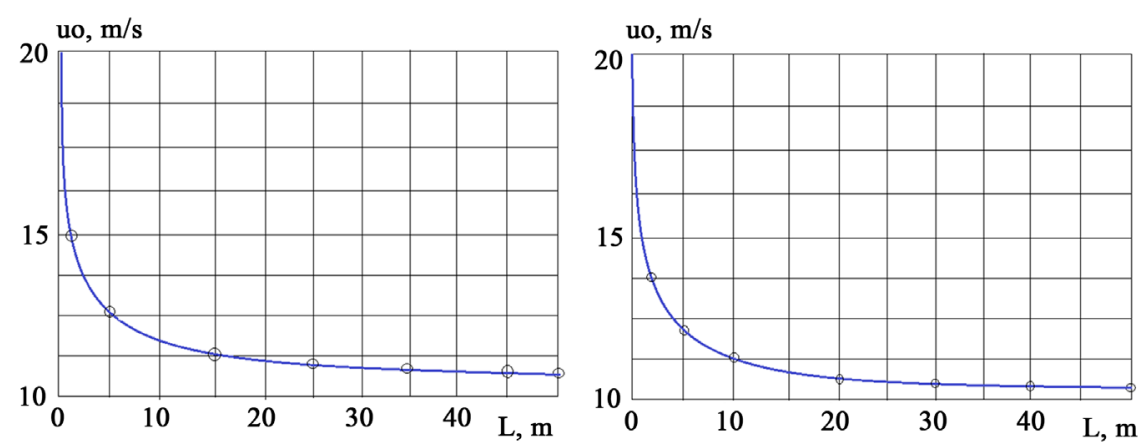

Figure 3 . The change in air velocity along the length of the pipeline.
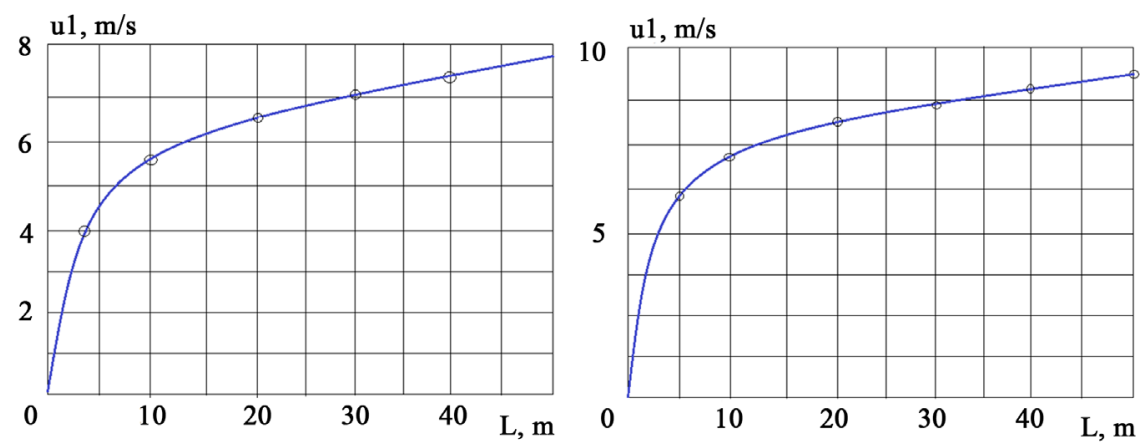

Figure 4. Change the speed of cotton along the length of the pipeline.

there is no significant change in speed. At large values of the interaction coefficient, the nature of the change of parameters is preserved. The difference is only the intensity of the change. Figure 5 represents the change in the porosity of cotton in both cases at a distance of $50 \mathrm{~m}$ reaches the same value, $0.75-0.80$.

Only with a large $k$, the growth of porosity in the initial $10-20$ meters is much stronger and when it reaches $30 \mathrm{~m}$, the change is significantly stabilized. A, the change in pressure, on the contrary (Figure 6), with smaller $\mathrm{k}$ decreases more strongly than relatively large $k$. This shows that $k$ negatively affects the resistance force, i.e., with its increase, the resistance force decreases, and with decreasing, on the contrary, it increases.

In both cases (Figure 7) and values, the air velocity shows a character of decline with almost the same intensity. Only if in the first graphs the stabilization of air velocity occurs at a value of $9-10 \mathrm{~m} / \mathrm{s}$, then in the following graphs, i.e., at large values, the air velocity remains without changes after the value of $15 \mathrm{~m} / \mathrm{s}$.

The speed of cotton (Figure 8) also changes according to the previous pattern: at smaller values $k_{n}$ the intensity of the increase in speed is relatively low, but this increase is maintained for a long time, and at large values $k_{n}$ the growth rate is much higher, but after 30 meters the speed of cotton is almost stabilized. At the same time, the speed is about $9-10 \mathrm{~m} / \mathrm{s}$. If you pay attention to the air speed, you can see its value-at a distance of $40-50$ meters it is about $14-15 \mathrm{~m} / \mathrm{s}$, that is, the air is ahead of cotton with a relative speed equal to the difference of their speeds. The ratio of cotton velocity to air velocity will give the latency coefficient, which in our case is equal to the tabular values of $k=0.7-0.75$ [8]. This 

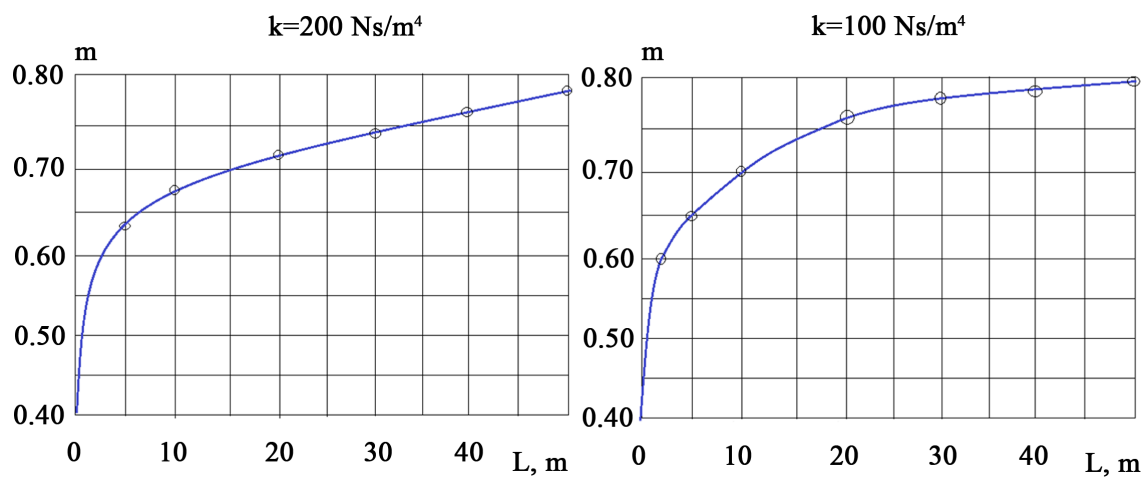

Figure 5. Changing the porosity of cotton along of the pipeline.
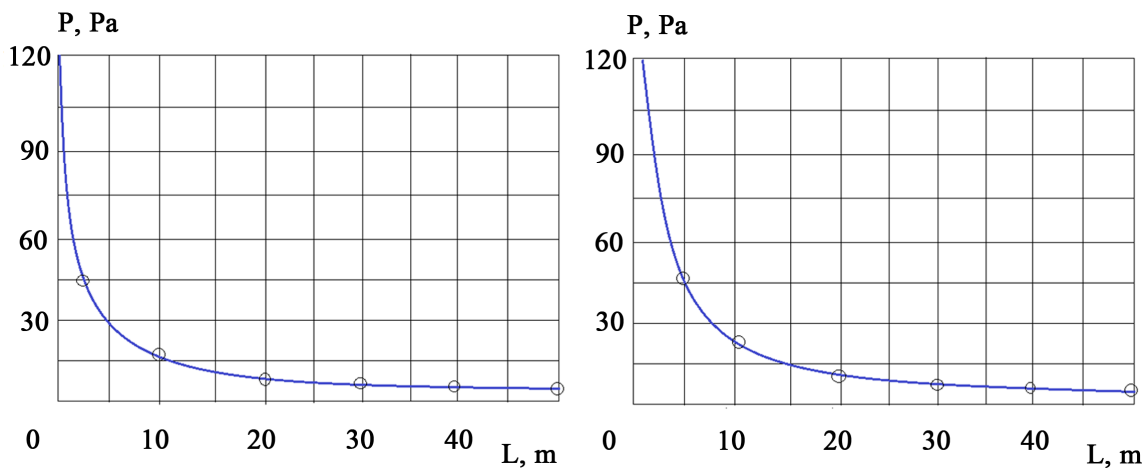

Figure 6. The change in air pressure along the length of the pipeline.
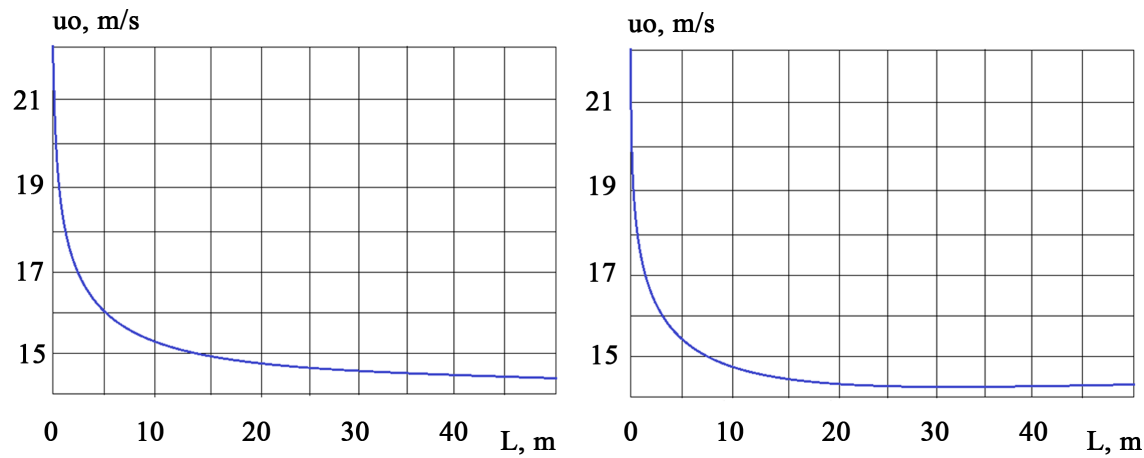

Figure 7. The change in air velocity along the length of the pipeline.
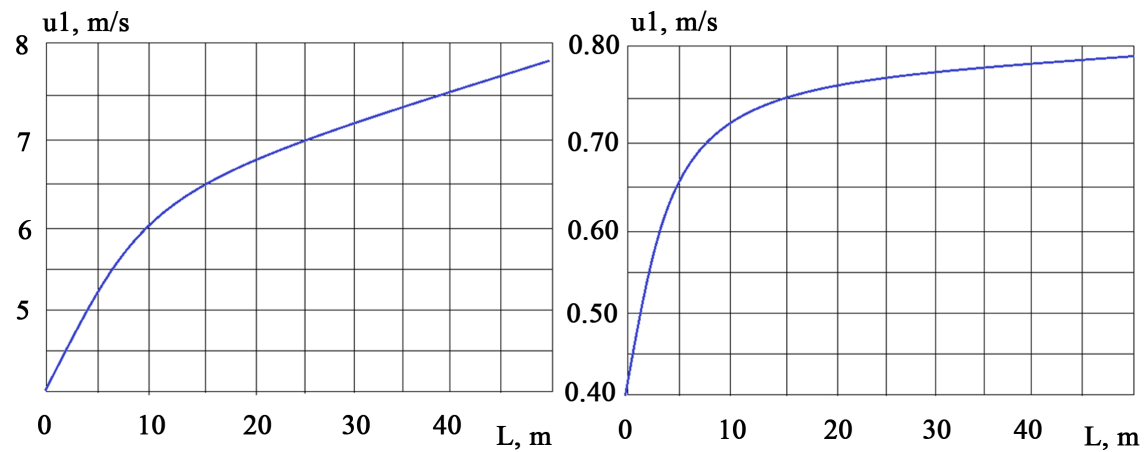

Figure 8. Change the speed of cotton along the length of the pipeline. 
ratio shows how late cotton is from air. The fact of the equality of theoretical values with the table values, which are established experimentally, proves the compliance of the established theoretical pattern with the natural pattern of movement of the cotton-air mixture in the transport pipelines of pneumatic installations and will provide an opportunity to offer the established formulas for the calculation of pneumatic transport and its design [11].

The resulting model is universal and can be used for calculations of pipelines with variable cross-section.

\section{Findings}

1) Research has established that during pneumatic transportation, in the initial 20 - 25 meters of the pipeline, the porosity of transported cotton greatly increases, which indicates its exposure to tensile deformation and proves that cotton is loosened, fluffed, and trash is separated from it.

2) The entry of cotton into the pneumatic conveying pipeline leads to a sharp decrease in pressure and air velocity, and the speed of cotton in the initial 15 to 20 meters of the pipeline increases strongly, then with more moderate intensity.

3) The multi-speed model of the movement of a cotton-air mixture as a heterogeneous medium in pneumotransport pipelines more correctly describes the process of moving air and material during pneumatic transportation, which makes it possible to suggest its use for calculating pneumatic transport and in its design.

\section{Conflicts of Interest}

The authors declare no conflicts of interest regarding the publication of this paper.

\section{References}

[1] Mardanov, B. and Sarimsakov, O. (2017) Theoretical Study of the Process of Mechanical Breakdown of Cotton Seeds. Journale of Fergana Politechniall I, No. 1, 125-127.

[2] Abbazov, I., Sarimsakov, O., Khodjiev, M. and Mardonov, B. (2018) Waste Produced at Cotton Waste Factories. American Journal of ASCIT Communications, 5, 22-28.

[3] Sarimsakov, O. and Ruzmetov, M. (2016) Dynamics of the Interaction of the Worker Organs with Cotton in the Bulletproof Machine. Journal of Mechanical Problems, No. 4, 31-34.

[4] Abdukarimovich, M.O., Ibragimovich, A.K. and Sharipjanovich, S.O. (2018) Designing a New Design of a Loading Cylinder for Pneumomechanical Spinning Machines. Engineering, 10, 345-356. https://doi.org/10.4236/eng.2018.106025

[5] Kholmirzaev, F., Azimov, S., Abdurahimov, K. and Sarimsakov, O. (2019) Investigation of the Loss of Air Pressure in the Pipeline of the Cotton Pneumatic Conveying. Saudi Journal of Engineering and Technology Dubai, United Arab Emirates, 4, 23-27.

[6] Muradov, R., Burnashev, R.Z. and Sarimsakov, O.Sh. (2001) The Dynamic Task of 
the Interaction of Raw Cotton with the Working Bodies of Processing Machines. Journal of Mechanic Problems, No. 34, 55-58.

[7] Obidov, A., Akhmedkhodjaev, Kh., Sarimsakov, O. and Holikov, Q. (2018) Investigation of the Properties of Fibrous Cotton Seeds, for Sorting on a Mesh Surface. The Journal of Engineering, 10, 572-578. https://doi.org/10.4236/eng.2018.109041

[8] Sarimsakov, O. (2016) The Possibility of Reducing Cotton Consumption in Cotton. American Journal of Science and Technology, 4, 68-72.

http://www.aascit.org/journal/et

[9] Sarimsakov, O. (2019) Improving the Process of Filing Cotton in Pneumatic Transport and Its Transportation by Air. "Navruz" Publishing House, Namangan, 252.

[10] Sarimsakov, O. and Gaybnazarov, E. (2016) About Energy Consumption in Pneumatic Conveying of Raw Cotton. American Journal of Energy and Power Engineering, 3, 26-29.

[11] Sarimsakov, O., Xusanov, C. and Muradov, R. (2016) The Change in Air Pressure Along the Length of the Pipeline Installation for Pneumatic Conveying of Raw Cotton. Journal of Engineering and Technology, 3, 89-92.

http://www.aascit.org/journal/et 